\title{
The Combination Neuroprotective Abilities of Resveratrol and Naringenin in Attenuation of Sleep Deprivation Complications in Rats
}

\author{
Zahra Abbasy $^{1}$, Hesam Adin Atashi², Felicia Agatha ${ }^{3}$, Fatemeh Mirparsa ${ }^{4}$, Hamid Zaferani Arani ${ }^{2 凶}$, Amirhossein $^{2}$ \\ Gaeini ${ }^{5}$, Atousa Hashemi ${ }^{6}$ \\ 'Faculty of Medicine, Kashan University of Medical Sciences, Kashan, Iran \\ ${ }^{2}$ Young Researchers and Elite Club, Islamic Azad University, Tehran Medical Sciences, Tehran, Iran. \\ ${ }^{3}$ Senior High School Student, Tzu Chi Secondary School PIK, DKI Jakarta, Indonesia \\ ${ }^{4}$ Midwifery Undergraduate and Master Student of Health Services Management, Department of Health Care Management, Faculty \\ of Health, Tehran Medical Sciences, Islamic Azad University, Tehran, Iran \\ 5 Department of Experimental Medicine (DIMES), University of Genoa, Genoa, Italy \\ ${ }^{6}$ Department of Molecular Medicine, University of Padua, Padua, Italy
}

\begin{abstract}
Background: Sleep loss is one of the most important health problems in the world, and about 30 to 40 percent of ordinary people suffer from it. This study aimed to investigate the neuroprotective effects of the combination of resveratrol and naringenin in attenuation of sleep deprivation (SD) complications in rats. Materials and Methods: In this experimental study, 72 Wistar male rats were randomly divided into three main groups, including control, sham, and 7-days SD group. Each of its main groups consisted of three subgroups, including without drug, vehicle, and combination therapy groups (naringenin [100 mg/ $\mathrm{kg}$ ], resveratrol [100 $\mathrm{mg} / \mathrm{kg}])$. The day after the latest injection, the fear conditioning memory tests, locomotor activity test, hot plate, and forced swimming tests (FST) were carried out on all rats, and then sham and SD groups were induced 48 hours of non-REM SD (device off and on, respectively) and these behavioral tests were repeated for all rats again. Finally, the brains of all rats were removed and histopathologically examined, and stained with nissl and TUNNEL. Results: To assess fear condition memory, the rate of latency to first freezing in the visual and auditory phase increased in sham and SD rats that received vehicle or no drug $(\mathrm{P}<0.001)$, which indicates memory corruption. Injection of the combination of naringenin and resveratrol reduced the latency to first freezing $(\mathrm{P}<0.001)$, which means improved memory. In the FST test, injection of naringenin and resveratrol reduced the rate of immobility $(\mathrm{P}<0.001)$, which means improved depressive behavior. The naringenin and resveratrol reduced the pain perception threshold. Also, the naringenin and resveratrol reduced apoptosis compared to the control and vehicle groups $(\mathrm{P}<0.001)$. Conclusions: The combination of naringenin and resveratrol compared to other groups could improve memory and mood as well as reduce apoptosis, depression,
\end{abstract}

and pain perception threshold. [GMJ.2021;10:e2315] DOI:10.31661/gmj.v10i0.2315

Keywords: Combination Effects; Resveratrol; Naringenin; Sleep Deprivation

\section{GMJ}

Copyright(C) 2021, Galen Medical Journal. This is an open-access article distributed under the terms of the Creative Commons Attribution 4.0 Internationa License (http://creativecommons.org/licenses/by/4.0/) Email:info@gmj.ir $\triangle$ Correspondence to:

Hamid Zaferani Arani; Young Researchers and Elite Club, Tehran Medical Sciences, Islamic Azad University, Attarimoqaddam Ave, Haqani Ave, Dr. Shariati St, Tehran, Iran, P. O. BOX: 19168-93813, Tehran, IranTelephone Number:+9821-22006660

Email Address: hamid_zaferani73@yahoo.com 


\section{Introduction}

Sleep deprivation (SD) and insomnia have been recognized as one of the most important health problems for many years [1] and refer to cases in which a person suffers from one or more of one of the problems such as insomnia, frequent awakenings during sleep at night, complain of drowsiness during the day or abnormal movements, and moods and feelings during sleep [2]. According to published reports, approximately $40 \%$ of people in the world suffer from sleep disorders [3]. SD can lead to the impaired perception of pain, memory impairments, and depression [3, 4]. Studies have shown that disrupting the physiological order of sleep and depriving it, depending on the duration and type of deprivation, causes consequences such as stress, pathological anxiety in humans, the imbalance between oxidative substances produced and cleansing by the antioxidant defense system, and oxidative damage causes $[4,5]$. Numerous studies have also shown that SD can increase the risk of high blood pressure, obesity, diabetes, depression, heart attacks, and strokes [5-7]. In addition, many studies have shown that insomnia can also reduce the power of concentration and consciousness [6]. SD can also negatively affect other aspects of a person's life and cause work errors in various jobs [7]. Studies have shown that the levels of proteins and enzymes involved in the Krebs cycle change following SD [8]. Some animal studies have shown that SD impairs hippocampaldependent memory and emotional memory and increases anxiety $[9,10]$. Naringenin is a primary flavonoid (a crystalline and colorless derivative of flavonoids) found abundantly in tangerines, oranges, and grapefruits [11]. In recent years, naringenin and resveratrol have been considered due to their many medicinal properties $[11,12]$.

In addition, naringenin is very important as an antioxidant and lowers blood cholesterol factor [13].

Naringenin and resveratrol can manage and regulate chronic and acute inflammatory responses $[14,15]$. In a study, it was stated that naringenin and resveratrol have several properties, including anti-inflammatory, antioxidant, antitumor, cardiovascular protection, reducing metabolic problems (such as diabetes), and may affect the neuronal systems as well as the sleep process [16, 17]. Arafah et al. indicated that compounds in citrus fruits have several properties, including their sedative effects [18]. Also, in an experimental study, it was found that naringenin has antihypertensive effects that control seizures, and this rate is equivalent to the effects of sodium valproate, which is mainly due to its effect on potassium and calcium channels [19]. Leenaars et al. [20] showed that SD for two days increased immobility in Wistar rats in forced swimming tests (FST) and induced depressive-like behavior. Therefore, this study aimed to investigate the neuroprotective effects of naringenin and resveratrol combination on attenuating total SD complications in rats.

\section{Materials and Methods}

\section{Animals}

Seventy-two male Wistar rats (weighing 220 to $240 \mathrm{~g}$ ) were prepared from the Pharmacology Department, Tehran University of Medical Sciences (Tehran, Iran). The animals were kept in standard laboratory conditions at $22 \pm 3{ }^{\circ} \mathrm{C}$.

The light and dark cycle was established as 12 hours of light and 12 hours of darkness. Each animal was tested only once, and each experimental group consisted of eight animals. During the experiments, sufficient water and food were provided to the animals. Behavioral tests were also performed in the light cycle.

This study has been approved by the ethics committee of Tehran Islamic Azad University of Medical Sciences (codes: IR.IAU. TMU.REC.1399.373 and IR.IAU.TMU. REC.1399.412), and all measures taken on laboratory animals have been approved by this committee based on ethical protocols.

\section{Study Design}

Rats were randomly divided into three main groups, including control, sham, and SD. Each group was divided into subgroups, including 
without drug, vehicle, and combination therapy. The control group consisted of normal rats receiving no medication. The vehicle group received only $0.25 \%$ carboxymethylcellulose solution (Sigma Aldrich, Germany) with $0.9 \%$ saline or rapamycin intraperitoneally (IP) daily for seven days. The drug group was given a combination of naringenin and resveratrol IP for seven days at a dose of $100 \mathrm{mg} / \mathrm{kg}$ [21, 22] dissolved in $0.25 \%$ carboxymethylcellulose and $0.9 \%$ saline daily. The control group only had a water and food diet for seven days. In the sham sleep group, rats were placed in the SD device turned off for 48 hours for exposure to environmental stress alone, and in the SD group, rats were placed in the SD device turned on. The minimum number of rats in each of these subgroups was eight. Then, the day after the last injection (the eighth day), fear condition memory tests (train with shock four times), locomotor activity test, hot plate, and FST were performed on all rats. All rats were deprived of 48 hours of nonREM sleep except for the control group, and these behavioral tests were done in all rats again, then the effect of the combination of naringenin and resveratrol on the behavior of rats before and after SD was investigated. Finally, the brains of all rats were removed and histopathologically examined, and stained with nissl and TUNNEL to define the apoptosis rate.

\section{Naringenin and Resveratrol Preparation}

These drugs were prepared in powder form (Sigma-Aldrich, Germany) with 98\% purity. In the acute toxicity studies, the median lethal dose (LD50) of naringenin and resveratrol was reported as $600 \mathrm{mg} / \mathrm{kg}$ [23].

\section{SD Device and Model}

The SD model was performed less than five hours after the memory test session. SD was performed by a digital device with the ability to adjust various parameters so that the animal was placed on a pedal, and according to the timing that was adjusted on the device, the pedals moved and alerted the animal before the pedal entered the water, they would go on the next pedal. This process caused the animal to have amnesia. It should be noted that the napping model was set and performed on the same device [24].

The laboratory animal was placed on an SD machine for 48 hours. The animal was given enough time to receive water and food.

\section{Conditional Fear Memory Learning Device}

In this study, the fear memory conditioning device measured fear memory. The device consisted of a box mounted on the top of the camera box for viewing the animal and two speakers for playing audio. These speakers were connected to the computer on that the audio player program was installed. A 24-watt light bulb was installed in the corner of the box. The conditioning box was placed inside the acoustic box. At the bottom of the box were parallel steel rods attached to the electric shock device. After each use of the device, the device's floor was cleaned with water and alcohol [25].

The purpose of using this device was to measure the rate of memory of visual and auditory fear in rats. The protocol used for this device in this study consisted of three sessions, one session for training and two sessions for testing.

\section{Conditional Fear Training and Assessment}

In the first session, which was related to training, a black space was provided for the rats, and first, the rat was inserted for 150 seconds, and after 150 seconds, four consecutive shocks were given at the end of the sound, with a distance of 30 seconds between each shock and after the last shock and sound, waited for 30 seconds and then the rat was removed. The shock intensity was 1 ma with a frequency of $50 \mathrm{~Hz}$ for five seconds. This step took approximately 6.5 minutes.

In the second session (visual fear memory test), the rat was placed in the black space for five minutes without entering any stimuli. The latency to freezing and freezing time of the rat was recorded in terms of seconds, and other behaviors of the rat, such as self-decoration and standing on two legs were recorded in terms of time. Finally, its rate was expressed as a percentage. In the third session (auditory memory test), the rat was placed in a white space, and first, the rat was placed in the box for 
150 seconds, then the sound was played four times in a row without shock, with 30 seconds between each sound played, and after the last sound, it was waited for 30 seconds, and then the rat was removed. It should be noted that the light conditions in this experiment were constant. Freezing time was equivalent to fear memory, so the longer it was, the less likely it would be damaged [25].

\section{FST}

In this study, the FST was performed to evaluate the depressed mood in the studied animal in five minutes. This test was performed in a clear cylindrical container. The cylinder filled with water by $2 / 3$ of its volume, and the rat would float slowly in the water. Using a chronometer, the time of each laboratory animal activity was measured over a time of five minutes. The duration of immobility or inactivity was equated with depressed mood, which was expressed as a percentage. Activities such as swimming and jumping to get out of the cylinder were also recorded [26].

\section{Movement System Test (Locomotor Activity Test / Open Field)}

The laboratory animal was examined using an open field to ensure the motor health of the rat. To perform this test, rats were taken to the laboratory one hour before the test to familiarize themselves with the environment. Then each rat was allowed to move freely inside the box for one minute before the test to familiarize itself with the test box. Each rat was then left in the box for five minutes to move. Meanwhile, it was tracked through a rat movement tracking system.

This test aimed to measure the motor activity of the animal based on the change from its previous position to a new position inside the device, which was measured by optical eyes installed on the sides of the floor of the device. After adjusting the device and placing the animal in its center, the number of movement activities in the monitor that was connected to the device was displayed in five minutes.

Measurable parameters by this device include the number of linear movements, stereotyped movements, and the number of lifting movements on the legs that were lifted on the hind legs. Of these indicators, the lower frame analyzed the number of linear movements and the number of stereotyped movements, and the upper frame analyzed and counted the number of lifting movements on the legs [27].

\section{Hot Plate Test}

To reduce the stress of placing rats in the hot plate machine, before turning on the machine by placing each rat 3-4 times at intervals of 3-5 minutes on the plate of the device, we tried to acquaint the rat with the tests plate environment. The rat was then placed on a hot plate set at $54^{\circ} \mathrm{C}$ for this test. The time interval between placing the rat on the device and the time of licking the animal's foot was considered as the time for the pain to reach the threshold by heat.

The test did not last more than 30 seconds to prevent tissue damage [28].

\section{Staining}

Nissl staining was used for the identification morphology and pathology of neurons and neural tissues. Also, terminal deoxynucleotidyl transferase dUTP nick end labeling (TUNNEL) as a modern alternative to analyzing the formation of DNA fragments for apoptosis identification [29].

\section{Statistical Analysis}

GraphPad Prism 5.0 software was used for the statistical analysis of data. The results of the present study were adjusted in the behavioral section. One- and two-way ANOVA, as well as post hoc Tukey tests were used for comparison between groups. Also, a significance level of $\mathrm{P}<0.05$ has been considered.

\section{Results}

\section{Latency to First Freezing in the Auditory Stage}

The results showed that there was a statistically significant difference between control groups $(\mathrm{F} 2.21=237.03$ and $\mathrm{P}<0.001)$.

Also, it showed that the latency to first freezing increased in rats that received a vehicle $(\mathrm{P}<0.001)$, which means memory destruction. On the other hand, injecting a combination of 
naringenin $(100 \mathrm{mg} / \mathrm{kg})$ and resveratrol (100 $\mathrm{mg} / \mathrm{kg}$ ) reduced the latency to first freezing $(\mathrm{P}<0.001)$, which means improved memory. Also, there was a statistically significant difference between the control and the sham groups (sleep $[\mathrm{F} 1.42=370.45, \mathrm{P}<0.001]$, drugs $[\mathrm{F} 2.42=305.89, \mathrm{P}<0.001]$, and the effect of SD and drug [F2.42 = 29.2, P<0.001]. The latency to first freezing increased in the without drug rats of the sham groups $(\mathrm{P}<0.001)$, which means memory impairment. Also, our results indicated that there was a statistically significant difference between the sleep and SD groups (sleep $[\mathrm{F} 1.42=3.85$, $\mathrm{P}<0.05]$, drug [F2.42 = 423.83, $\mathrm{P}<0.001])$. In contrast, SD and drug groups $(\mathrm{F} 2.42=1.68$, $\mathrm{P}>0.05$ ) were not significant. Also, our results showed that the rate of delay until the first freezing increased in the without drug group of $\mathrm{SD}(\mathrm{P}<0.001)$. The combination of naringenin and resveratrol significantly reduced the latency to first freezing (Figure-1A, P<0.001). Latency to First Freezing in the Visual (Contextual) Stage.

The results showed that there was a statistically significant difference between the control groups (with normal sleep; $\mathrm{F} 2.21=1069.73, \mathrm{P}<0.001)$. The latency to first freezing increased in rats that received the vehicle $(\mathrm{P}<0.001)$, which means memory impairment. On the other hand, injection of a combination of naringenin and resveratrol reduced the latency to first freezing $(\mathrm{P}<0.05)$, which means improved memory.

The results showed that there was a statistically significant difference between the control groups (with normal sleep) and sham groups (sleep $[\mathrm{F} 1.42=1648.02, \mathrm{P}<0.001]$, drug $[\mathrm{F} 2.42=55.19, \mathrm{P}=0.001]$, and $\mathrm{SD}$ and drug $[\mathrm{F} 2.42=197.8, \mathrm{P}<0.001])$. Also, the latency to first freezing was increased in the without drug group of the sham $(\mathrm{P}<0.001)$ and in rats
A

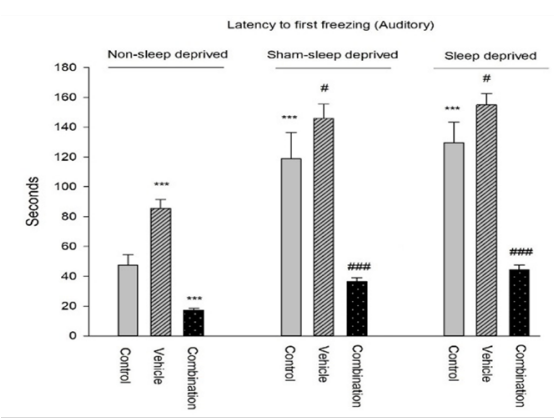

C

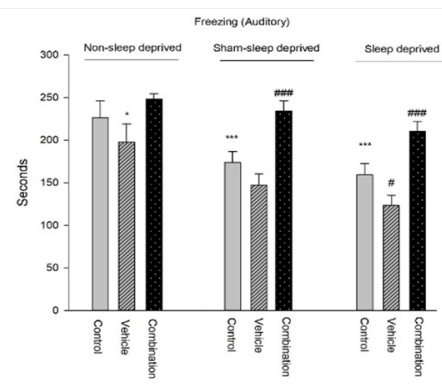

B

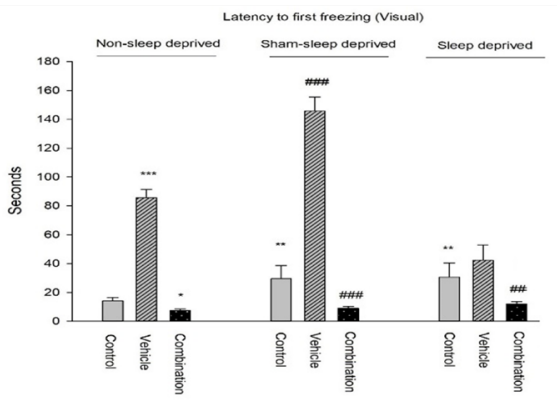

D



Figure 1. A: Latency to first freezing in the auditory stage in rats in groups with normal sleep ( 3 columns left), sham groups ( 3 middle columns), and SD groups (3 right columns) with combination of naringenin and resveratrol. ${ }^{* \star *} P<0.001,{ }^{* *} P<0.01$, and ${ }^{*} P<0.05$ compared with the no drug group of normal sleep; \#\# P <0.01, \#\# P <0.00,1 and \# P <0.05, compared with the related control groups. B: Latency to first freezing in the visual phase in rats in the groups with normal sleep ( 3 columns left), sham groups ( 3 middle columns), and SD groups ( 3 columns on the right) with the combination of naringenin and resveratrol. ${ }^{* *} \mathrm{P}<0.001,{ }^{* *} \mathrm{P}<0.01$, and ${ }^{*} \mathrm{P}<0.05$ compared with the no drug group of normal sleep; \#\# P<0.01, \#\# P<0.001, and \# $\mathrm{P}<0.05$ compared with the related control group. C: The time of freezing in the auditory stage in rats in the groups with normal sleep ( 3 columns left), sham groups ( 3 middle columns), and SD groups ( 3 columns on the right) that received the combination of naringenin and resveratrol. ${ }^{* *} P<0.001,{ }^{* *} P<0.01$, and ${ }^{*} P<0.05$ compared with the no drug group of normal sleep; \#\# $P<0.01$, \#\# P $<0.001$, and \# $P<0.05$ compared with the related control group. D: Visual freezing time in rats in the normal sleep group (3 columns left), sham groups (3 middle columns), and SD groups (3 right columns) that received a combination of naringenin and resveratrol. ${ }^{* * *} \mathrm{P}<0.001$, ${ }^{* *} \mathrm{P}<0.01$, and ${ }^{*} \mathrm{P}<0.05$ compared with the no drug group of normal sleep; \#\# $P<0.01$, \#\# $P<0.001$, and \# $P<0.05$, compared with the related control group. 
that received the vehicle $(\mathrm{P}<0.001)$, which means memory impairment.

There was a statistically significant difference between the sham and SD groups (sleep $\quad[\mathrm{F} 1.42=202.71, \quad \mathrm{P}<0.001]$, drug $[\mathrm{F} 2.42=468.17, \mathrm{P}<0.001]$, and the effect of $\mathrm{SD}$ and drug $(\mathrm{F} 2.42=227.11, \mathrm{P}<0.001)$. Also, the results showed the latency to first freezing increased in without drug group of SD $(\mathrm{P}<0.001)$, which means memory impairment (Figure-1B).

\section{Freezing Time in the Auditory Stage}

The results showed that there was a statistically significant difference between control groups $(\mathrm{F} 2.21=14.06, \mathrm{P}<0.001)$. Also, the freezing time decreased in rats that received vehicles $(\mathrm{P}<0.05)$, which means memory impairment. The results showed that there was a statistically significant difference between control (with normal sleep) and sham groups (sleep $[\mathrm{F} 1.42=70.61, \quad \mathrm{P}<0.001]$, drug $[\mathrm{P}<0.001$, $\mathrm{F} 2.42=76.55$,], and effect of SD and drug ( $\mathrm{F} 2.42=9.61, \mathrm{P}<0.05])$. Also, the freezing time in the without drug rats of the sham group decreased $(\mathrm{P}<0.001)$, which means memory impairment. There was a statistically significant difference between sham and SD groups (sleep $[\mathrm{F} 1.42=33, \mathrm{P}<0.001$ ), drug $[F 2.42=202.88, \mathrm{P}<0.001]$, and the effect of $\mathrm{SD}$ and drug $[\mathrm{F} 2.42=0.72, \mathrm{P}>0.05])$. Also, freezing time decreased in without drug group of SD $(\mathrm{P}<0.001)$, which means memory impairment (Figure-1C). The combination of naringenin and resveratrol significantly increased the freezing time $(\mathrm{P}<0.001)$.

\section{Freezing Time in the Visual Stage}

Freezing time decreased in the without drug of the sham group $(\mathrm{P}<0.001)$, which indicate memory impairment. There was a statistically significant difference between sham and SD groups (sleep [F1.42=15.93, $\mathrm{P}<0.001]$, drug $[\mathrm{F} 2.42=942.76, \mathrm{P}<0.001]$, and the effect of $\mathrm{SD}$ and drug $(\mathrm{F} 2.42=4.12, \mathrm{P}<0.05])$. Also, the freezing time in the without drug of the SD group decreased $(\mathrm{P}<0.001)$, which indicates memory impairment (Figure-1D). The combination of naringenin and resveratrol significantly increased the freezing time $(\mathrm{P}<0.001)$.

\section{Time of Immobility in the FST}

There was a statistically significant difference between the control groups (F2.21=140.47, $\mathrm{P}<0.001)$. The time of immobility increased in rats receiving the vehicle $(\mathrm{P}<0.001)$. The immobility time increased in the without drug of the sham group $(\mathrm{P}<0.001)$, which means an increase in depressive behavior. The time of immobility also increased in rats that received the vehicle $(\mathrm{P}<0.001)$, which means an increase in depressive behavior. On the other hand, injecting the combination of naringenin and resveratrol reduced immobility $(\mathrm{P}<0.001)$, which means improved depressive behavior (Figure-2A).

\section{Climbing Time in FST}

There was a statistically significant difference between the data of control and sham groups (sleep [F1.42=78.76, $\mathrm{P}<0.001]$, drug $[\mathrm{F} 2.42=404.87, \mathrm{P}<0.001]$, and effect of SD and drug $[\mathrm{P}<0.001, \mathrm{~F} 2.42=44.51])$. Also, the time of climbing in the without drug of the sham group decreased $(\mathrm{P}<0.001)$. The vehicle also reduced the climbing $(\mathrm{P}<0.001)$. On the other hand, the combination of naringenin and resveratrol $(\mathrm{P}<0.001)$ increased the climbing, which indicates a decrease in depressive behavior (Figure-2B).

\section{Swimming in FST}

The results showed that there was a statistically significant difference between control (with normal sleep) and sham groups (sleep [F1.42=19.58, $\mathrm{P}<0.001]$, and drug $[\mathrm{F} 2.42=22.72, \mathrm{P}<0.001])$. While the effect of $\mathrm{SD}$ and drug $(\mathrm{F} 2.42=1.89, \mathrm{P}>0.05)$ was not significant. Also, the time of swimming following injection of a combination naringenin and resveratrol decreased in rats in the sleep sham group (Figure-2C, $\mathrm{P}<0.01$ ).

\section{Pain Threshold Level}

The results showed that there was a statistically significant difference between the sham and $\mathrm{SD}$ groups (sleep $[\mathrm{F} 1.42=11.39, \mathrm{P}<0.01]$, drug $[\mathrm{F} 2.42=61.6, \mathrm{P}<0.001]$, and the effect of $\mathrm{SD}$ and drug $[\mathrm{F} 2.42=13.36, \mathrm{P}<0.001])$ was not significant. Also, the pain threshold increased in the without drug group of SD 

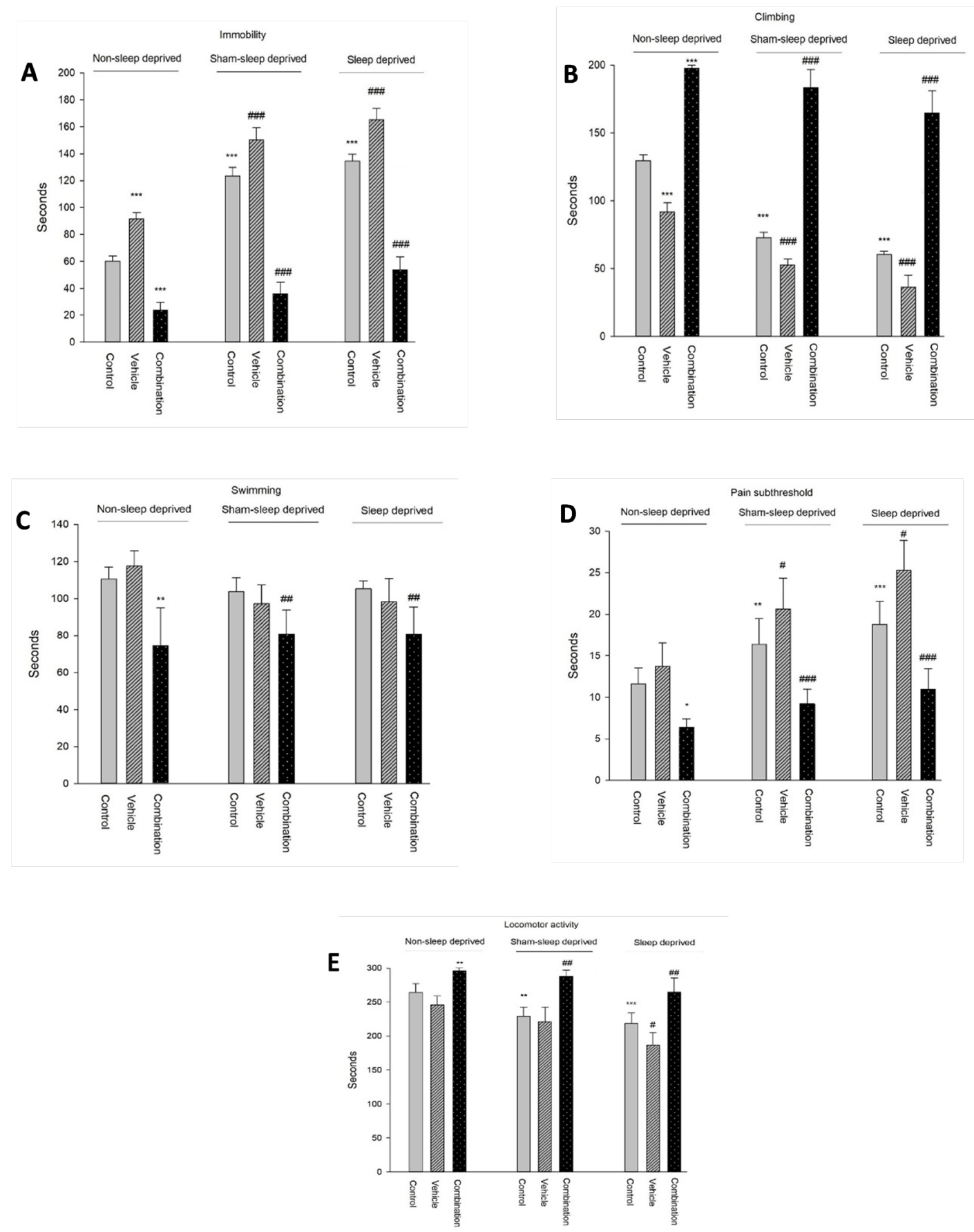

Figure 2. A: The time of immobility in rats in the groups with normal sleep (left 3 columns), sham groups ( 3 middle columns), and SD groups ( 3 right columns) that receiving combination of naringenin and resveratrol. ${ }^{* * *} \mathrm{P}<0.001$, ${ }^{* *} \mathrm{P}<0.01$, and ${ }^{*} \mathrm{P}<0.05$ compared with the no drug group of normal sleep; \#\# $\mathrm{P}<0.01$, \#\# P $<0.001$, and \# $\mathrm{P}<0.05$ compared with the related control group. B: The time of climbing in rats in the groups with normal sleep ( 3 columns on the left), sham groups ( 3 in the middle column), and SD groups ( 3 columns on the right) that received a combination of naringenin and resveratrol. ${ }^{* *} \mathrm{P}<0.001$, ${ }^{* *} \mathrm{P}<0.01$, and ${ }^{*} \mathrm{P}<0.05$ compared with the no drug group of normal sleep; \#\# P $<0.01$, \#\# P $<0.001$, and \# P $<0.05$ compared with the related control group. C: The time of swimming in rats in the groups with normal sleep (left 3 columns), sham groups ( 3 middle columns), and SD groups ( 3 right columns) that received a combination of naringenin and resveratrol. ${ }^{* * *} P<0.001$, ${ }^{* *} P<0.01$, and ${ }^{*} P<0.05$ compared with the no drug group of normal sleep; \#\# P $<0.01$, \#\# P $<0.001$, and \# P<0.05 compared with the related control group. D: Pain thresholds in rats in the normal sleep group ( 3 columns left), sham groups ( 3 middle columns), and SD groups ( 3 right columns) that received a combination of naringenin and resveratrol. ${ }^{* * *} \mathrm{P}<0.001$, ${ }^{* *} \mathrm{P}<0.01$, and ${ }^{*} \mathrm{P}<0.05$ compared with the no drug group of normal sleep; \#\# $\mathrm{P}<0.01$, \#\# $\mathrm{P}<0.001$, and \# $\mathrm{P}<0.05$ compared with the related control group. E: Locomotor activity in rats in the groups with normal sleep ( 3 columns left), sham groups (3 middle columns), and SD groups ( 3 right columns) that received a combination of naringenin and resveratrol. ${ }^{* * *} P<0.001,{ }^{* *} P<0.01$, and ${ }^{*} \mathrm{P}<0.05$ compared with the no drug group of normal sleep. \#\# $\mathrm{P}<0.01$, \#\#\# $\mathrm{P}<0.001$ and \# $\mathrm{P}<0.05$ compared with the related control group. 
rats $(\mathrm{P}<0.001)$. The vehicle also increased the pain threshold in the sham group $(\mathrm{P}<0.05)$. On the other hand, combination naringenin and resveratrol reduced the pain threshold (Figure-2D, $\mathrm{P}<0.001$ ).

\section{Locomotor Activity}

The results showed that there is a statistically significant difference between the data of sham and SD groups (drug $[\mathrm{F} 2.42=17.86, \mathrm{P}<0.001]$ and effect of SD and drug [F2.42 $=18.79$, $\mathrm{P}<0.001]$ ). While the effect of sleep ( $\mathrm{F} 1.42=0.01, \mathrm{P}>0.05)$ was not significant. Also, the locomotor activity in the without drug group of the SD decreased $(\mathrm{P}<0.001)$. Also, the vehicle reduced locomotor activity in sham rats $(\mathrm{P}<0.05)$. On the other hand, locomotor activity increased in rats receiving a combination of naringenin and resveratrol $(\mathrm{P}<0.01)$, which indicates increasing the activity of rats (Figure-2E).

\section{Nissl and TUNEL Staining Assessment}

Nissl staining showed the presence of dark neurons, significantly more in sham and vehicle groups. In groups of naringenin and resveratrol combination, significantly apoptosis decreased compared to other groups (Figure-3). TUNEL staining showed the DNA fragments for apoptosis comparatively more in sham and vehicle groups. TUNNEL staining, confirmed by nissl staining, showed that apoptosis was significantly reduced in the drug group (Figure-4).

\section{Discussion}

Our results showed that SD impairs memory function. The pain threshold following injection of the combination naringenin and resveratrol was reduced. Locomotor activity of sham and SD rats was reduced. Indeed, the combination of naringenin and resveratrol increased locomotor activity. Also, the combination of naringenin and resveratrol compared to other groups improves memory and mood, reduces apoptosis, immobility, depression, and pain perception threshold. In the past, studies have been designed and performed to evaluate the effect of naringenin on memory function. Liaquat et al. shown that naringenin improves learning and memory retention by improving the function of antioxidant enzymes [30]. In our study, there was also memory impairment in sham groups. Sham group was designed to evaluate the effect of stress on memory function. So we can conclude that stress has also damaged memory. On the other hand, stress can increase inflammation and oxidative stress. Previous studies have shown that chronic stress increases markers of oxidative stress [31]. Another study showed that mild unpredictable stress in Wistar rats increased inflammation and oxidative stress [32]. Increased inflammation and oxidative stress could impair cognitive activity; hence, memory function was impaired in sham sleep rats in our study. Also, the similarity of the performance of vehicle rats to sham and SD rats could be due to injection-induced stress.

\section{A}
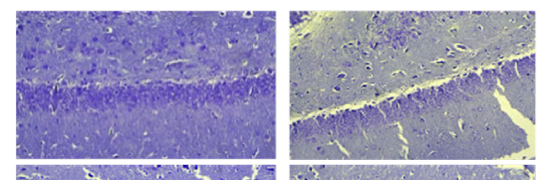

B
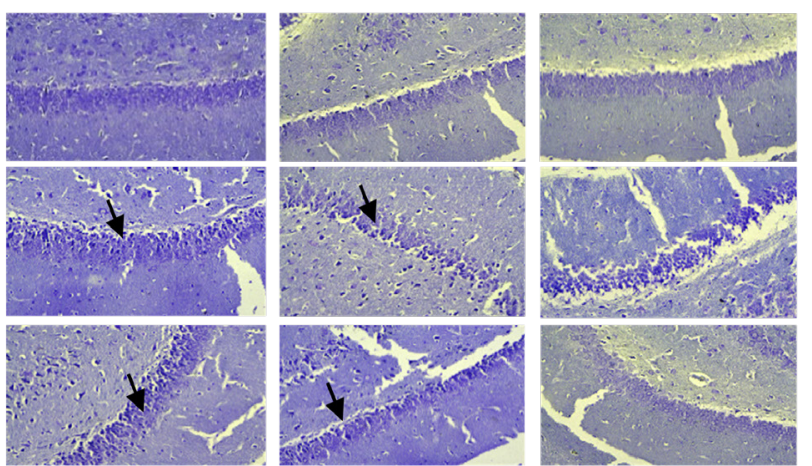

C

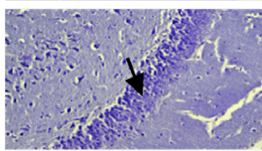

Figure 3. Nissl staining rats' hippocampus. Demonstration micrographs of each study group. A: the hippocampus of control animals did not contain any damaged neurons; B: Sham groups; C: SD groups. The hippocampus of rats in the sham without drug and vehicle (B) and SD without drug and vehicle $(\mathrm{C})$ groups were characterized by fewer Nissl stain neurons (thin arrows) than that in the combination of naringenin and resveratrol group, and markedly contained shrunken, intensely stained, and dystrophic neurons (thick arrows). Treatment rats with naringenin and resveratrol $(C)$ significantly reduced neuronal cell loss in the hippocampus (magnification $\times 10$ ). 


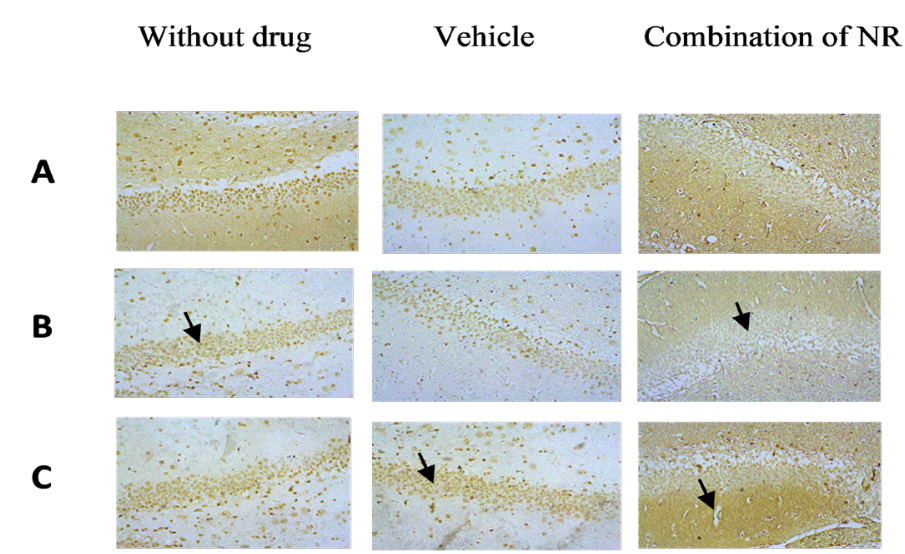

Figure 4. TUNNEL staining of rats' hippocampus. Demonstration micrographs of each study group. A: The hippocampus of control animals did not contain any damaged neurons; B: Sham groups; C: SD groups. The hippocampus of rats in the sham without drug and vehicle $(B)$ and SD without drug and vehicle $(C)$ groups were characterized by fewer TUNNEL stain neurons (thin arrows) than that in the combination of naringenin and resveratrol group, and markedly contained shrunken, intensely stained, and dystrophic neurons (thick arrows). Treatment rats with naringenin and resveratrol $(C)$ significantly reduced neuronal cell loss in the hippocampus (magnification $\times 10)$.

Due to the neuroprotective effects of the combination of naringenin and resveratrol, it has been suggested that this substance may potentially improve memory function. Naringenin has potent effects in increasing antioxidant activity and reducing oxidative stress [33].

Hua et al. shown that naringenin significantly improves learning capacity and memory retention by reducing the expression of genes involved in apoptosis (such as Bax and $\mathrm{Bad}$ ), and reducing inflammatory factors such as interleukin-6 [34]. Naringenin also improves learning and memory performance in Alzheimer's rats [35]. In rats with chronic unpredictable stress, naringenin also improves spatial memory function [36].

Complete deprivation of sleep for 24 hours causes depressive behavior and increases immobility of Wistar rats in FST [37]. Previous research has shown that deprivation of REM sleep for five days induces depressive behavior in rats [38]. The results of the current study are in line with previous studies. Another important point is that the stress of SD alone can have a devastating effect on mood. Our results showed that rats in the vehicle and the sham groups also had depressive behavior (increased immobility time).

Therefore, as mentioned in the memory section, stress could cause depression by inflammation and increased apoptosis, and decreased BDNF levels. On the other hand, our results showed that a combination of naringenin and resveratrol improved depressive behavior in sham and SD rats. Previous studies have reported the antidepressant effects of naringenin [39].

As our data showed, the pain threshold was elevated in rats in SD groups. Also, the combination of naringenin and resveratrol reduced this threshold in rats. So far, many studies showed the effect of SD or restriction on pain perception. The results of these studies have been somewhat contradictory, meaning that in some studies the pain threshold decreased, and in some studies, the pain threshold increased. For example, a previous study showed that chronic SD increased pain perception by increasing oxidative stress and inflammatory factors in the cortex and striatum [40].

Contrary to the results of these studies, two studies have shown that 24 hours of complete SD increases the pain threshold in Wistar rats $[41,42]$. In our study, SD increased the pain perception threshold. To find the possible mechanism of such an effect, we can refer to adenosine. Adenosine increases and decreases with sleep following SD and prolonged waking [42]. Previous studies have shown that adenosine has an analgesic effect [43]. In our study, the increase in pain threshold following SD could be due to the involvement of adenosine. 


\section{Conclusion}

Our results showed that SD impairs memory function. Reducing thepainthreshold following injection of the combination naringenin and resveratrol; however, no definite mechanism was determined. Locomotor activity of sham and SD rats was reduced. Indeed, the combination of naringenin and resveratrol increased locomotor activity. Locomotor activity did not affect memory function because in the sham and SD groups, freezing decreased, while in rats with the combination naringenin and resveratrol, the freezing time has increased. Also, the combination of naringenin and resveratrol compared to other groups improves memory and mood, as well as reduction in apoptosis, immobility, depression, and pain perception threshold.

\section{Conflicts of Interest}

All authors declare that there is no conflict of interest.

\section{References}

1. Libman E, Fichten C, Creti L, Conrod K, Tran DL, Grad R, et al. Refreshing sleep and sleep continuity determine perceived sleep quality. Sleep Disord. 2016;2016:7170610.

2. Nikfar B, Moazzami B, Chaichian S, Ghalichi L, Ekhlasi-Hundrieser M, Chashmyazdan M, et al. Sleep Quality and its Main Determinants Among Staff in a Persian Private Hospital. Arch Iran Med. 2018;21(11):524-9.

3. Eide PK, Vinje V, Pripp AH, Mardal KA, Ringstad G. Sleep deprivation impairs molecular clearance from the human brain. Brain. 2021;144(3):863-74.

4. Liew SC, Aung T. Sleep deprivation and its association with diseases-a review. Sleep Med. 2021;77:192-204.

5. Alimohammadzadeh $\mathrm{K}$, Akhlaghdoust M, Bahrainian S A, Mirzaei A. Survey on Mental Health of Iranian Medical Students: A Cross- sectional Study in Islamic Azad University, Shiraz E-Med J. 2017;18(7):e14929.

6. Cross N, Paquola C, Pomares FB, Perrault AA, Jegou A, Nguyen A, et al. Cortical gradients of functional connectivity are robust to state-dependent changes following sleep deprivation. Neuroimage. 2021;226:117547.

7. Cherubini JM, Cheng JL, Williams JS, MacDonald MJ. Sleep deprivation and endothelial function: reconciling seminal evidence with recent perspectives. Am J Physiol Heart Circ. 2021;320(1):29-35.

8. Chai Y, Fang Z, Yang FN, Xu S, Deng
Y, Raine A, et al. Two nights of recovery sleep restores hippocampal connectivity but not episodic memory after total sleep deprivation. Sci Rep. 2020;10(1):1-1.

9. Bishir M, Bhat A, Essa MM, Ekpo O, Ihunwo AO, Veeraraghavan VP, et al. Sleep deprivation and neurological disorders. Biomed Res Int. 2020; 2020:5764017.

10. Vishwakarma LC, Sharma B, Singh V, Jaryal AK, Mallick HN. Acute sleep deprivation elevates brain and body temperature in rats. J Sleep Res. 2021;30(2):e13030.

11. Joshi R, Kulkarni YA, Wairkar S. Pharmacokinetic, pharmacodynamic and formulations aspects of Naringenin: An update. Life sci. 2018;215:43-56.

12. Fourny N, Lan C, Sérée E, Bernard M, Desrois M. Protective effect of resveratrol against ischemia-reperfusion injury via enhanced high energy compounds and eNOS-SIRT1 expression in type 2 diabetic female rat heart. Nutrients. 2019;11(1):105.

13. Tutunchi H, Naeini F, Ostadrahimi A, Hosseinzadeh-Attar MJ. Naringenin, a flavanone with antiviral and antiinflammatory effects: A promising treatment strategy against COVID-19. Phytother Res. 2020;34(12):3137-47.

14. Zeng W, Jin L, Zhang F, Zhang C, Liang W. Naringenin as a potential immunomodulator in therapeutics. Pharmacol Res. 2018;135:122-6.

15. Said RS, Mantawy EM, El-Demerdash 
E. Mechanistic perspective of protective effects of resveratrol against cisplatininduced ovarian injury in rats: emphasis on anti-inflammatory and anti-apoptotic effects. Naunyn Schmiedebergs Arch Pharmacol. 2019;392(10):1225-38.

16. Clementi N, Scagnolari C, D'Amore A, Palombi F, Criscuolo E, Frasca F, et al. Naringenin is a powerful inhibitor of SARS-CoV-2 infection in vitro. Pharmacol Res. 2020; 163:105255.

17. Meng T, Xiao D, Muhammed A, Deng J, Chen L, He J. Anti-inflammatory action and mechanisms of resveratrol. Molecules. 2021;26(1):229.

18. Arafah A, Rehman MU, Mir TM, Wali AF, Ali R, Qamar W, et al. Multitherapeutic potential of naringenin (4', 5, 7-trihydroxyflavonone): experimental evidence and mechanisms. Plants (Basel). 2020;9(12):1784.

19. Chin LH, Hon CM, Chellappan DK, Chellian J, Madheswaran T, Zeeshan F, et al. Molecular mechanisms of action of naringenin in chronic airway diseases. Eur J Pharmacol. 2020;879:173139.

20. Leenaars CH, Joosten RN, Zwart A, Sandberg H, Ruimschotel E, Hanegraaf MA, et al. Switch-task performance in rats is disturbed by $12 \mathrm{~h}$ of sleep deprivation but not by $12 \mathrm{~h}$ of sleep fragmentation. Sleep. 2012;35(2):211-21.

21. Khajevand-Khazaei MR, Ziaee P, Motevalizadeh SA, Rohani M, AfshinMajd S, Baluchnejadmojarad $\mathrm{T}$, et al. Naringenin ameliorates learning and memory impairment following systemic lipopolysaccharide challenge in the rat. Eur J Pharmacol. 2018;826:114-22.

22. Singh N, Agrawal M, Doré S. Neuroprotective properties and mechanisms of resveratrol in in vitro and in vivo experimental cerebral stroke models. ACS Chem Neurosci. 2013;4(8):1151-62.

23. Raj J, Chandra M, Dogra TD, Pahuja M, Raina A. Determination of median lethal dose of combination of endosulfan and cypermethrin in wistar rat. Toxicol Int. 2013;20(1):1.

24. Leenaars $\mathrm{CH}$, Joosten RN, Zwart A, Sandberg H, Ruimschotel E, Hanegraaf MA, et al. Switch-task performance in rats is disturbed by $12 \mathrm{~h}$ of sleep deprivation but not by $12 \mathrm{~h}$ of sleep fragmentation. Sleep. 2012;35(2):211-21.

25. Campbell TL, Kochli DE, McDaniel MA, Myers MK, Dunn ME, Diana VA, et al. Using Extinction-Renewal to Circumvent the Memory Strength Boundary Condition in Fear Memory Reconsolidation. Brain Sci. 2021;11(8):1023.

26. Moreno-Santos B, Marchi-Coelho C, Costa-Ferreira W, Crestani CC. Angiotensinergic receptors in the medial amygdaloid nucleus differently modulate behavioral responses in the elevated plusmaze and forced swimming test in rats. Behav Brain Res. 2021;397:112947.

27. Hazim AI, Ramanathan S, Parthasarathy S, Muzaimi M, Mansor SM. Anxiolyticlike effects of mitragynine in the openfield and elevated plus-maze tests in rats. J Physiol Sci. 2014;64(3):161-9.

28. Bai Y, Peng W, Yang C, Zou W, Liu $\mathrm{M}, \mathrm{Wu} \mathrm{H}$, et al. Pharmacokinetics and metabolism of naringin and active metabolite naringenin in rats, dogs, humans, and the differences between species. Front Pharmacol. 2020;11:364.

29. Radmansouri M, Ghiasvand S, Shahriari F, Aryan H, Jangholi E, Javidi MA. Hypericin Induces Apoptosis in MDAMB-175-VII Cells in Lower Dose Compared to MDA-MB-231. Archives of Iranian Medicine. 2018;21(9):387-92.

30. Liaquat L, Batool Z, Sadir S, Rafiq S, Shahzad S, Perveen T, et al. Naringenininduced enhanced antioxidant defence system meliorates cholinergic neurotransmission and consolidates memory in male rats. Life Sci. 2018;194:213-23.

31. Liao D, Lv C, Cao L, Yao D, Wu Y, Long $\mathrm{M}$, et al. Curcumin Attenuates Chronic Unpredictable Mild StressInduced Depressive-Like Behaviors via Restoring Changes in Oxidative Stress and the Activation of Nrf2 Signaling Pathway in Rats. Oxid Med Cell Longev. 2020;2020:9268083.

32. Guo Y, Gan X, Zhou H, Zhou H, Pu S, Long $X$, et al. Fingolimod suppressed the chronic unpredictable mild stress-induced depressive-like behaviors via affecting 
microglial and NLRP3 inflammasome activation. Life Sci. 2020;263:118582.

33. Khan H, Ullah H, Tundis R, Belwal T, Devkota H, Daglia $M$, et al. Dietary Flavonoids in the Management of Huntington's Disease: Mechanism and Clinical Perspective. eFood. 2020;1(1):38-52.

34. Hua FZ, Ying J, Zhang J, Wang XF, Hu $\mathrm{YH}$, Liang YP, et al. Naringenin pretreatment inhibits neuroapoptosis and ameliorates cognitive impairment in rats exposed to isoflurane anesthesia by regulating the PI3/Akt/PTEN signalling pathway and suppressing NF-kappaBmediated inflammation. Int $\mathrm{J}$ Mol Med. 2016;38(4):1271-80.

35. Ghofrani S, Joghataei MT, Mohseni $\mathrm{S}$, Baluchnejadmojarad $\mathrm{T}$, Bagheri $\mathrm{M}$, Khamse $\mathrm{S}$, et al. Naringenin improves learning and memory in an Alzheimer's disease rat model: Insights into the underlying mechanisms. Eur J Pharmacol. 2015;764:195-201.

36. Tayyab M, Farheen S, M MMP, Khanam N, Mobarak Hossain M, Shahi MH. Antidepressant and Neuroprotective Effects of Naringenin via Sonic Hedgehog-GLI1 Cell Signaling Pathway in a Rat Model of Chronic Unpredictable Mild Stress. Neuromolecular Med. 2019;21(3):250-61.

37. Rezaie M, Nasehi M, Vaseghi S, Alimohammadzadeh $\mathrm{K}$, Islami Vaghar M, Mohammadi-Mahdiabadi-Hasani $\mathrm{MH}$, et al. The interaction effect of sleep deprivation and cannabinoid type 1 receptor in the CA1 hippocampal region on passive avoidance memory, depressivelike behavior and locomotor activity in rats. Behav Brain Res. 2021;396:112901.

38. Chen L, Wang Z, Wang XM. Rapid eyemovement sleep for five days deprivation causes delayed depressive-like behavior in mice. South Med J. 2016;36(5):660-4.

39. Yi LT, Liu BB, Li J, Luo L, Liu Q, Geng $\mathrm{D}$, et al. BDNF signaling is necessary for the antidepressant-like effect of naringenin. Prog Neuropsychopharmacol Biol Psychiatry. 2014; 48:135-41.

40. Arora S, Venugopalan A, Dharavath RN, Bishnoi M, Kondepudi KK, Chopra
K. Naringenin Ameliorates Chronic Sleep Deprivation-Induced Pain via Sirtuin 1 Inhibition. Neurochem Res. 2021;46(5):1177-87.

41. Javad-Moosavi BZ, Nasehi M, Vaseghi S, Jamaldini SH, Zarrindast MR. Activation and Inactivation of Nicotinic Receptnors in the Dorsal Hippocampal Region Restored Negative Effects of Total (TSD) and REM Sleep Deprivation (RSD) on Memory Acquisition, Locomotor Activity and Pain Perception. Neuroscience. 2020;433:200-11.

42. Eydipour Z, Nasehi M, Vaseghi S, Jamaldini SH, Zarrindast MR. The role of 5-HT4 serotonin receptors in the CA1 hippocampal region on memory acquisition impairment induced by total (TSD) and REM sleep deprivation (RSD). Physiol Behav. 2020;215:112788.

43. Eisenach JC, Hood DD, Curry R, Sawynok J, Yaksh TL, Li X. Intrathecal but not intravenous opioids release adenosine from the spinal cord. J Pain. 2004;5(1):64-8. 